\title{
An Algorithm of the Time-delay Alignment Based on Iterative Estimation
}

\author{
Li Yang ${ }^{1, a}$, Zhang Xiao-yi ${ }^{1, b}$, Hu Yun-peng ${ }^{1, c}$,Shen Zhi-xiang ${ }^{1, d}$ and \\ Zhao Biao ${ }^{2, e}$ \\ ( ${ }^{1}$ Zhengzhou Information Science and Technology Institute, Zhengzhou, China, 450002 \\ ${ }^{2}$ National Defense Information Institute, Wuhan, 430010) \\ a15514512618@126.com, btx19900910@126.com, ${ }^{\mathrm{c}}$ xiangwenyao@sina.com, ${ }^{\mathrm{d}}$ chengb_cn@163.co \\ $\mathrm{m},{ }^{\mathrm{e}} 478482969 @ q q . c o m$
}

Keywords: time-delay alignment; uniform antenna array; iterative estimation

\begin{abstract}
A novel algorithm aligning the time-delay of each signal received by a uniform antenna array is presented. In order to solve the problem that time-delay alignment performance is poor at the low signal-to-noise ratio (SNR) value, the proposed algorithm makes use of iterative estimation. Then we analyze and compare the performance between the proposed method and the time-delay alignment algorithm based on quasi synthesis reference (TAQSR), indicating that the new algorithm has better performance. Simulation results show that the new algorithm not only improves the time-delay alignment performance in the multi-antenna signal synthesis system, especially in low SNR, but is also free from the actual value of the time-delay difference, compared with TAQSR.
\end{abstract}

\section{Introduction}

TDOA estimation has always been an important problem in the multi-antenna signal synthesis system. The multi-antenna signal synthesis technology ${ }^{[1]}$ is the use of multiple antennas to receive the same signal, by weighting every antenna signal to acquire a synthesized signal, a quality improved synthesized signal can be achieved. Because there may be many differences between the antenna signals, multi-antenna synthesis system must estimate and compensate the differences of parameters. In the multi-antenna signal synthesis system, TDOA estimation is actually a time-delay alignment problem between each received signal and the reference signal. The approaches for TDOA between two signals include the basic cross-correlation method, the generalized cross-correlation method ${ }^{[2]}$, the higher-order cumulant method ${ }^{[3]}$, adaptive time-delay estimation algorithm ${ }^{[4]}$ and so on. The TDOA estimation performance mainly depends on SNR of received signal, observation time and signal bandwidth. In general case, the TDOA estimation performance between the two signals can't meet the need of time-delay alignment in the multi-antenna synthesis system. The research ${ }^{[5]}$ shows $^{2}$ that using the same information of the received signals can reduce the effect of noise on estimation performance and improve TDOA estimation performance in low SNR of the received signal in multi-antenna signal synthesis system.

In multi-antenna signal model, the ways to solve TDOA estimation become multi-channel cross-correlation method $^{[5]}$, eigenvalue decomposition method ${ }^{[6]}$, redundant fusion algorithm ${ }^{[7]}$, the time-delay alignment algorithm based on quasi synthesis reference (TAQSR) ${ }^{[8]}$ and so on. The multi-channel cross-correlation method and eigenvalue decomposition method are restricted by the antenna formations. The redundant fusion algorithm makes full use of the correlations of the estimated redundant time differences to improve estimation performance; however it is not easy to calculate the weight yet. For each signal, TAQSR regards synthesized signal of the other antennas as the reference signal, to increase the SNR of the reference signal and improve estimation performance. However, this method is limited to the value of the time-delay difference between the received signals. When the time-delay difference is large, the SNR of the reference synthesized signal is too low to provide correct estimation results, leading to its performance deterioration.

In this paper, we first put forward a time-delay alignment algorithm based on iterative estimation to improve the time-delay alignment performance in low SNR. Then the method is compared with Foundation project: the National Natural Science Foundation of China (Grant No.61201380). 
TAQSR. Theoretical analyses show that the estimation performance of the proposed method is better than the composite reference one. Moreover, the proposed method avoids the problem that the time-delay difference between the received signals is low.

This paper is organized as follows. In Section 2, we describe the signal model and put forward the new algorithm of time-delay alignment based on iterative estimation. The theoretical analyses of the method and the comparison one which is based on quasi composite reference are presented in Section 3 and numerical experiments are described in Section 4. Finally, some concluding remarks are drawn out in Section 5.

\section{New alignment method}

\subsection{Signal model}

Suppose that we have a uniform antenna array that consists of $N(N \geq 3)$ receiving antennas. Because of the arrival time-delay difference, the received signal $x_{i}(t)(i=1,2, \ldots, N)$ in the Gaussian channel can be expressed as

$$
x_{i}(t)=s\left(t-\tau_{i}\right)+n_{i}(t)
$$

Where $s(t)$ is the stationary base-band signal, $\tau_{i}$ represents arrival time-delay. Furthermore, the additive noise $n_{i}(t)$ is modelled by zero-mean Gaussian random variables with independent real and imaginary parts. The noise of each antenna is independent and is not related with the signal.

The basic correlation method is based on the theory that $s(t), n_{i}(t)$ and $n_{j}(t)$ are not correlative. Therefore, the cross-correlation function of the received signal is:

$$
R_{x_{i} x_{j}}(\tau)=R_{s s}\left(\tau-\left(\tau_{i}-\tau_{j}\right)\right)
$$

On account of the autocorrelation function $\left|R_{s s}\left(\tau-\left(\tau_{i}-\tau_{j}\right)\right)\right| \leq R_{s s}(0)$, we can get that time-delay estimation as

$$
\hat{\tau}_{i j}=\arg \max \left\{E\left[x_{i}(t+\tau) x_{j}^{*}(t)\right]\right\}
$$

In practice, the uncorrelated hypothesis between signal and noise, noise and noise is not completely satisfied under some conditions. On the other hand, correlation function is a mathematical sense strict statistical average. However, we can only use the limited time average instead of statistical average and this will seriously affect the estimation performance. So, time-delay estimation value can be expressed as

$$
\hat{\tau}_{i j}=\tau_{i j}+n_{i j}
$$

Where $\tau_{i j}$ is real time-delay difference and $n_{i j}$ is estimation error caused by the noise, $n_{i j}$ follows the gauss distribution ${ }^{[8]}$, namely $n_{i j} \sim N\left(0, \sigma_{i j}^{2}\right)$.

Any two signals of the $N$ signals are used to estimate time-delay difference by the basic cross-correlation method and we can obtain $N(N-1) / 2$ time-delay difference estimates. The vector of estimates are expressed as

$$
\left[\hat{\tau}_{12}, \hat{\tau}_{13}, \cdots, \hat{\tau}_{1 N}, \hat{\tau}_{23}, \cdots, \hat{\tau}_{(N-1) N}\right]
$$

Making use of the correlation between the time estimates, the reduced influence of noise and improved time-delay estimation performance can be achieved. 


\subsection{Proposed method}

In the multi-antenna signal synthesis system, time-delay estimation aims at time-delay alignment of all signals. We generally select a signal as the reference signal and then estimate the time-delay difference between the other signals and the reference one. the proposed algorithm doesn't choose the reference signal as the traditional methods do, however through the iterative estimation, each time-delay of all signals converges to weighted sum of the initial time-delay of all the signals.

Assume that time-delay compensation value of each signal is

$$
\begin{gathered}
\Delta \tau_{1}=\alpha \tau_{12}+\alpha \tau_{13}+\ldots+\alpha \tau_{1 N}+n_{1}^{1} \\
\vdots \\
\Delta \tau_{N}=\alpha \tau_{N 1}+\alpha \tau_{N 2}+\ldots+\alpha \tau_{N N-1}+n_{N}^{1}
\end{gathered}
$$

where $\tau_{i j}=\tau_{i}-\tau_{j}$, the following time-delay of each signal results in the first compensation for time-delay is:

$$
\begin{gathered}
\tau_{1}^{1}=\tau_{1}^{0}-\left(\alpha \tau_{12}+\alpha \tau_{13}+\ldots+\alpha \tau_{1 N}\right)+n_{1}^{1} \\
\vdots \\
\tau_{N}^{1}=\tau_{N}^{0}-\left(\alpha \tau_{N 1}+\alpha \tau_{N 2}+\ldots+\alpha \tau_{N N-1}\right)+n_{N}^{1}
\end{gathered}
$$

Taking $\tau_{i j}=\tau_{i}-\tau_{j}$ on the upper formula , we can obtain:

$$
\begin{gathered}
\tau_{1}^{1}=(1-(N-1) \alpha) \tau_{1}^{0}+\alpha \tau_{2}^{0}+\alpha \tau_{3}^{0}+\ldots+\alpha \tau_{N}^{0}+n_{1}^{1} \\
\vdots \\
\tau_{N}^{1}=\alpha \tau_{1}^{0}+\alpha \tau_{2}^{0}+\ldots+\alpha \tau_{N-1}^{0}+(1-(N-1) \alpha) \tau_{N}+n_{N}^{1}
\end{gathered}
$$

The model can be written in matrix form as $\left[\tau_{1}^{1}, \tau_{2}^{1}, \ldots, \tau_{N}^{1}\right]=A\left[\tau_{1}^{0}, \tau_{2}^{0}, \ldots, \tau_{N}^{0}\right]^{T}+\left[n_{1}, n_{2}, \ldots, n_{N}\right]$, where

$$
A=\left[\begin{array}{cccc}
1-(N-1) \alpha & \alpha & \ldots & \alpha \\
\alpha & 1-(N-1) \alpha & \ldots & \alpha \\
\vdots & \vdots & \ddots & \vdots \\
\alpha & \ldots & \ldots & 1-(N-1) \alpha
\end{array}\right]
$$

After $\mathrm{n}$ times iterations, the following time-delay of each signal can be expressed as

$$
\left[\tau_{1}^{n}, \tau_{2}^{n}, \ldots, \tau_{N}^{n}\right]=A^{n}\left[\tau_{1}^{0}, \tau_{2}^{0}, \ldots, \tau_{N}^{0}\right]+\left[n_{1}^{n}, n_{2}^{n}, \ldots, n_{N}^{n}\right]
$$

By analyzing Matrix $A$, we can find $A=B \Lambda B^{-1}$, where $\Lambda=\operatorname{diag}\{1,1-N \alpha, \ldots, 1-N \alpha\}$,

$$
B=\left[\begin{array}{cccc}
1 & 1 & \cdots & 1 \\
1 & -1 & \cdots & 0 \\
\vdots & \vdots & \ddots & \vdots \\
1 & 0 & \cdots & -1
\end{array}\right]
$$

Therefore, we can achieve $A^{n}=B \Lambda^{n} B^{-1}$ and $\Lambda^{n}$ can be given by

$$
\Lambda^{n}=\operatorname{diag}\left\{1,(1-N \alpha)^{n}, \ldots,(1-N \alpha)^{n}\right\}
$$

When $0<\alpha<2 / N$ and $\mathrm{n}$ is sufficiently large integers, we can obtain

$$
A^{n}=B \Lambda^{n} B^{-1}=\frac{1}{N}\left[\begin{array}{cccc}
1 & 1 & \cdots & 1 \\
1 & 1 & \cdots & 1 \\
\vdots & \vdots & \ddots & \vdots \\
1 & 1 & \cdots & 1
\end{array}\right]
$$

Taking the above formula on formula, $\tau_{i}^{n}$ can be given by

$$
\tau_{i}^{n}=\frac{1}{N} \sum_{j=1}^{N} \tau_{j}^{0}+n_{i}^{n}
$$

Therefore it can be seen that after $\mathrm{n}$ iterations the time-delay $\tau_{i}^{n}$ of each signal can be expressed as 
$\frac{1}{N} \sum_{j=1}^{N} \tau_{j}^{0}$ and we implement the delay-time alignment between multiple signals. By the equation, the smaller $|1-N \alpha|$ is, quicker the convergence can be achieved. Namely when $0<\alpha \leq 1 / N$ the smaller is $\alpha$, more slowly the convergence can be achieved and when $1 / N<\alpha<2 / N$ the smaller $\alpha$ is, quicker the convergence can be achieved. Therefore, when $\alpha=1 / N$, the delay-time of each signal converges most quickly through iterations. Meanwhile we can find that the time-delay center in this paper fits the time-delay center of TAQSR. The following section performs analyses and comparisons of the two algorithms.

\section{Performance analysis}

\subsection{The proposed algorithm}

To facilitate the performance comparison of the two methods, both methods choose the fastest convergence coefficient. The method in this paper select $\alpha=1 / N$, namely the delay-time of each signal converges through first iteration. Therefore, the time-delay difference between the time-delay of the first signal and the reference time-delay can be expressed as

$$
\hat{\tau}_{1 c}=\frac{1}{N} \sum_{i=2}^{N} \hat{\tau}_{1 i}=\frac{1}{N}\left(\sum_{i=2}^{N} \tau_{1 i}+\sum_{i=2}^{N} n_{1 i}\right)=\tau_{1 c}+n_{1 c}
$$

Where $n_{1 c}=\frac{1}{N} \sum_{i=2}^{N} n_{1 i}$, So the estimated mean square error (MSE) of the time-delay difference can be expressed as

$$
M S E_{1}=E\left[\left(\hat{\tau}_{1 c}-\tau_{1 c}\right)^{2}\right]=\frac{1}{N^{2}}\left(\sum_{i=2}^{N} \operatorname{conv}\left(n_{1 i}, n_{1 i}\right)+\sum_{i=2}^{N} \sum_{j=2, j \neq i}^{N} \operatorname{conv}\left(n_{1 i}, n_{1 j}\right)\right)
$$

The literature [7] shows that the covariance for deviation of the time-delay estimate can be defined as

$$
\operatorname{conv}\left(n_{i j}, n_{k l}\right)=\frac{2 \pi}{T} \cdot \frac{\int_{\omega_{B}} \omega^{2}\left|F_{i j}(\omega)\right|^{2}\left|F_{k l}(\omega)\right|^{2} G_{m}(i j ; k l) d \omega}{\int_{\omega_{B}} \omega^{2}\left|F_{i j}(\omega)\right|^{2} S(\omega) d \omega \cdot \int_{\omega_{B}} \omega^{2}\left|F_{k l}(\omega)\right|^{2} S(\omega) d \omega}
$$

Where

$$
\begin{aligned}
G_{m}(i j ; k l)= & \left(S(\omega)+N_{i}(\omega) \delta_{i k}\right)\left(S(\omega)+N_{j}(\omega) \delta_{j l}\right) \\
& -\left(S(\omega)+N_{i}(\omega) \delta_{i l}\right)\left(S(\omega)+N_{j}(\omega) \delta_{j k}\right)
\end{aligned}
$$

$T$ is signal observation time and $\omega_{B}$ is signal bandwidth. $F_{i j}(\omega)$ is the weighted function of

the time-delay estimation and if we adopt the basic cross-correlation method, we can acquire $F_{i j}=1 . S(\omega)$ is the average power of the source signal and $N_{i}(\omega)$ is the average power of the noise. If we adopt the basic cross-correlation method, we can obtain $F_{i j}=1$.

On account of that the SNR of each signal is the same, the covariance can be expressed as

$$
\operatorname{conv}\left(n_{1 i}, n_{1 j}\right)=\left\{\begin{array}{l}
\sigma_{12}^{2}, i=j \\
\frac{S N R}{2 S N R+1} \sigma_{12}^{2}, i \neq j
\end{array}\right.
$$

Taking the above formula on formula, we can obtain

$$
M S E_{1}=\frac{N-1}{N^{2}} \frac{N \cdot S N R+1}{2 S N R+1} \sigma_{12}^{2}
$$

\subsection{The time-delay alignment algorithm based on quasi composite reference}

Similarly, we also choose the estimation performance of time-delay difference between the first 
signal and the reference time-delay as statistics. The synthesis of the other signals except the first signal can be expressed as

$$
x_{c}(t)=\sum_{i=2}^{N} s\left(t-\tau_{i}\right)+\sum_{i=2}^{N} n_{i}(t)
$$

Assume that the synthesis is ideal, so $x_{c}(t)$ and $S N R_{c}$ can be given by $x_{c}(t)=s\left(t-\tau_{c}\right)+n_{c}(t)$, $S N R_{c}=(N-1) \cdot S N R$, where $n_{c}(t)=\frac{1}{N-1} \sum_{i=2}^{N} n_{i}(t), \quad \tau_{c}=\frac{1}{N-1} \sum_{i=2}^{N} \tau_{i}$ 。

We calculate the correlation between the first signal and the synthesis of the other signals and regard the maximum of the correlation function as time-delay estimation

$$
\hat{\tau}_{1 c}^{\prime}=\underset{\tau}{\arg \max }\left\{E\left[x_{1}(t+\tau) x_{c}^{*}(t)\right]\right\}
$$

We can get to know $\hat{\tau}_{1 c}^{\prime}=\tau_{1 c}^{\prime}+n_{1 c}$, where $\hat{\tau}_{1 c}^{\prime}$ is the actual time-delay difference between the first signal and the synthesis of the other signals and $n_{1 c}$ is the estimation error with the distribution of $n_{1 c} \sim N\left(0, \sigma_{1 c}^{2}\right)$. Because the delay-time of each signal converges to $\frac{1}{N} \sum_{j=1}^{N} \tau_{j}^{0}$ through first iteration when $\alpha=\frac{N-1}{N}$, the time-delay difference between the time-delay of the first signal and the reference time-delay is $\tau_{1 c}=\frac{N-1}{N} \tau_{1 c}^{\prime}$. The method of time delay on the mean square error (MSE) can be expressed as:

$$
M S E_{2}=E\left[\left(\hat{\tau}_{1 c}-\tau_{1 c}\right)^{2}\right]=E\left\{\left[\frac{N-1}{N}\left(\hat{\tau}_{1 c}^{\prime}-\tau_{1 c}^{\prime}\right)\right]^{2}\right\}=\left(\frac{N-1}{N}\right)^{2} \operatorname{conv}\left(n_{1 c}, n_{1 c}\right)
$$

Combining formula, we can obtain:

$$
\frac{\operatorname{conv}\left(n_{1 c}, n_{1 c}\right)}{\operatorname{conv}\left(n_{12}, n_{12}\right)}=\frac{1}{N-1} \frac{N \cdot S N R+1}{2 S N R+1}
$$

Therefore, the mean square error (MSE) can be expressed as

$$
M S E_{2}=\frac{N-1}{N^{2}} \frac{N \cdot S N R+1}{2 S N R+1} \sigma_{12}^{2}
$$

From the above, it can be concluded that when the synthesis of the synthesis reference signal is ideal, the equation holds as $M S E_{1}=M S E_{2}$. However, we can't realize the complete alignment between the signals in practice, as it is impossible to achieve the ideal synthesis. So the time-delay alignment performance in this paper is superior to TAQSR. Moreover, when the time-delay difference between the receiving signals is large in TAQSR, the SNR of the synthesis reference signal is inferior to the receiving signal and the algorithm may not be of convergence, deteriorating estimation performance. However the proposed algorithm is not limited to the value of the time-delay difference.

\section{Simulation results}

A series of Monte-Carlo simulations using MATLAB were conducted to study the characteristics of the proposed algorithm, where time-delay estimator adopts the basic cross-correlation method and lagrange interpolation method. The simulation conditions are as follows: the signal is BPSK, adopting the ascending cosine molding in which the roll-off factor is 0.3 , where sampling frequency is $200 \mathrm{~Hz}$, chip-rate is 50 Baud, carrier frequency is 0 and the number of code element is 1000 , temporarily not considering the frequency difference and phase difference between multiple signals. The normalized SNR of the receiving signal is $E_{b} / N_{0}=-5 d B \sim 5 d B$. Monte Carl methods are simulated, the number of which is 10000 . The horizontal axis is the normalized signal-to-noise ratio and the vertical axis is the normalized mean square error (MSE) in the graph, where the unit is $d B$. 
Experiment 1: The MSE curves of time-delay estimate error for different $\mathrm{N}$ are presented in Fig. 1. It shows the results of using 2, 4 and 8 sensors respectively. Considering that the time-delay centers under different antenna number are different, we select the performance of the time-delay difference between the first signal and the second signal as a statistical estimate, namely $\Delta \tau_{1}-\Delta \tau_{2}$. As clearly shown in Fig.1, with the increase of the number of antenna, the normalized mean square error (MSE) reduced gradually, the performance of time estimates is better, especially in lower SNR.

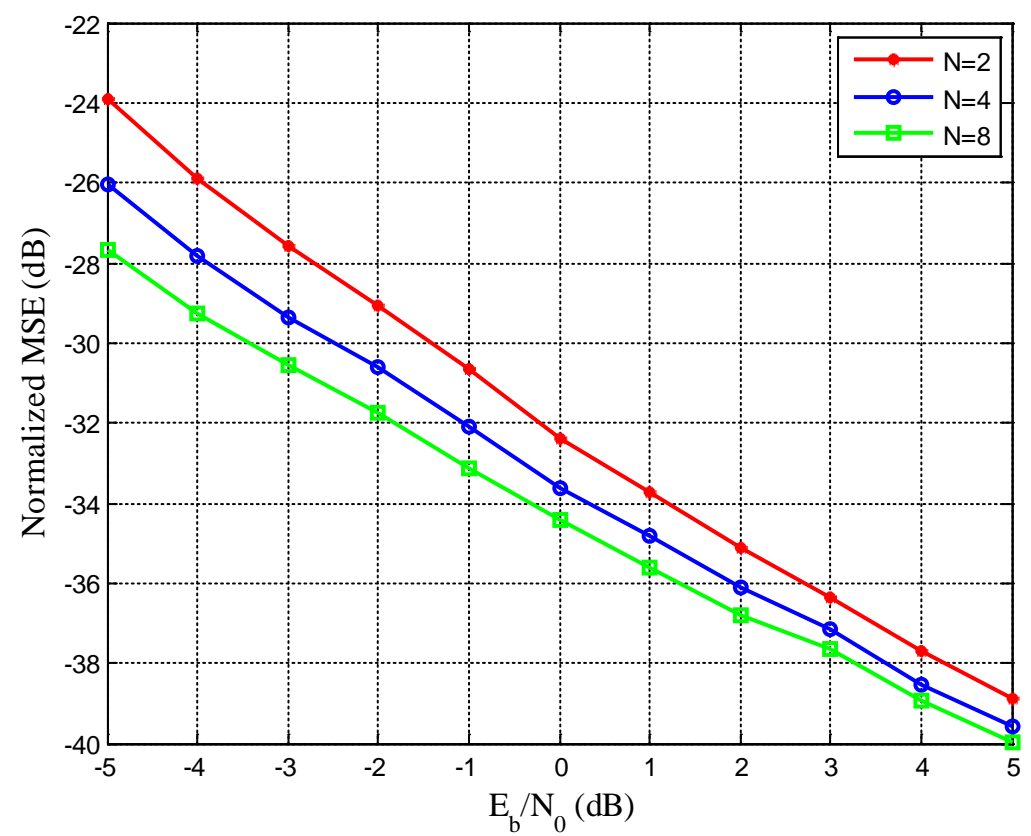

Fig.1 Estimation performance of different antenna number

Experiment 2: The algorithm is compared with TAQSR. We select the performance of the time-delay difference between the first signal and the reference time-delay. In Fig.2 the time-delay differences of multiple signals are randomly generated from $(-T \sim T)$ and in Fig.3 the time-delay differences of multiple signals are randomly generated from $(-2 T \sim 2 T)$. It can be seen from the Fig.2 and Fig.3, the algorithm is superior to the delay alignment algorithm based on synthetic reference , but in low SNR the inprovement is not obvious in Fig.2 and the inprovement is much obvious in Fig.3. Because the SNR of the reference signal in TAQSR is improved when the time-delay differences is little and the SNR of the reference signal is deteriorated when the time-delay differences is large. 


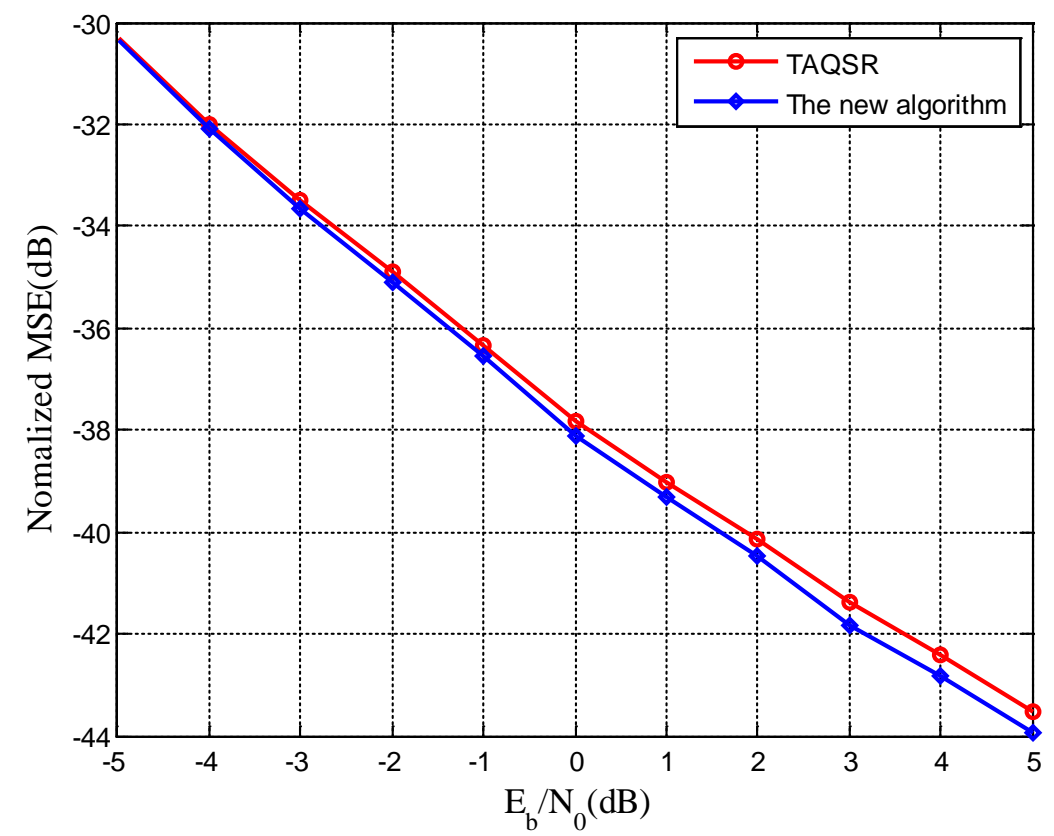

Fig.2 Comparison with TAQSR

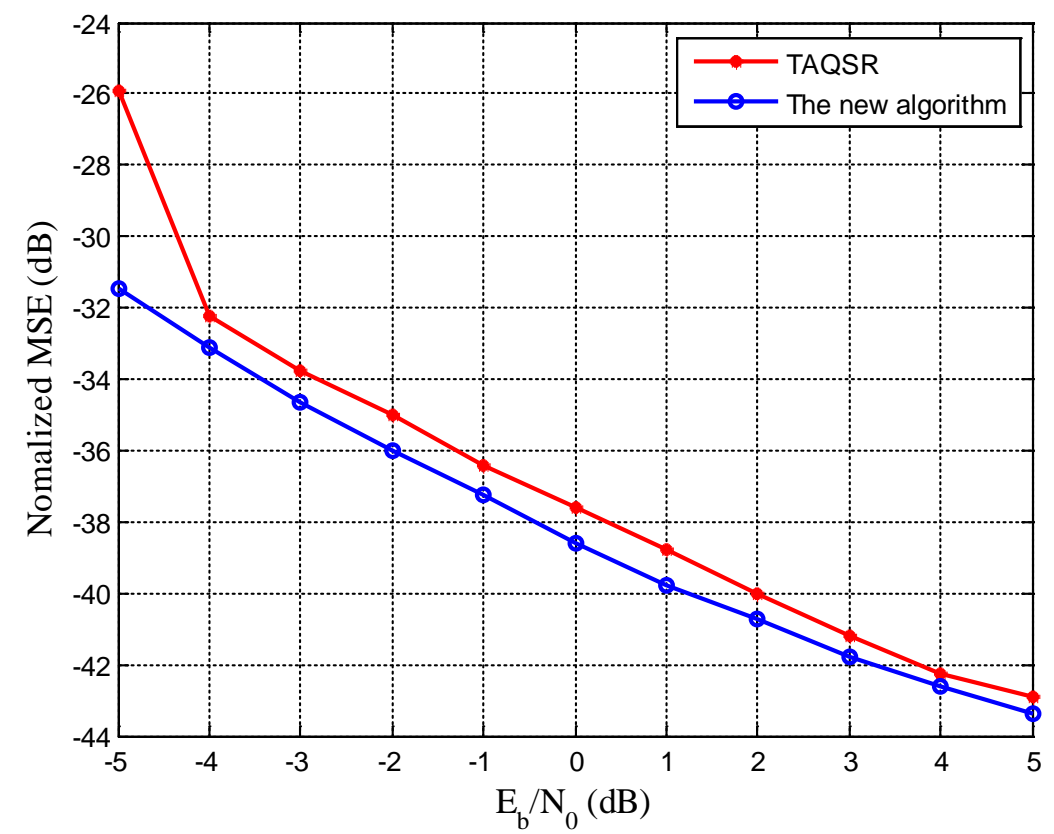

Fig.3 Comparison with TAQSR

\section{Conclusions}

This paper aims at the TDOA in the multi-antenna signal synthesis system and we put forward a new algorithm of time-delay alignment based on iterative estimation. The proposed method improves the time-delay estimation performance in low SNR. Moreover, we analyze and compare the performance between the new algorithm and TAQSR in theory, and the new algorithm extends the application as the application scope of $\mathrm{T}$ is limited to small value of time-delay . The result shows that the new algorithm improves the time-delay estimation performance in the multi-antenna signal synthesis system especially in low (SNR), and the performance in the new algorithm is superior to TAQSR, also the new algorithm in this paper is not limited to the size of the time-delay difference. 


\section{References}

[1] D.H.Rogstad, A.Mileant, and T. T. Pham, Antenna arraying techniques in the deep space network [M], Hoboken, New Jersey: John Wiley, 2003: 14-16.

[2] C.H.Knapp and G.C. Carter. The generalized correlation method for estimation of time delay [J]. IEEE Transactions on Acoustic, Speech and Signal Processing, 1976, 24(4): 320-327.

[3] J.K.Tugnait. Time delay estimation with unknown spatially correlated Gaussian noise [J]. IEEE Transactions on Signal Processing, 1993, 41(2): 549-558.

[4] Youn DH, Ahmed N, and G C. Carter. On using the LMS algorithm for time delay estimation [J]. IEEE Transactions on Acoustic, Speech and Signal Processing, 1982, 30 (5): 789-801.

[5] Jingdong Chen and Yiteng Huang. Robust time delay estimation exploiting redundancy among multiple microphones [J]. IEEE Transactions on Speech and Audio Processing, 2003, 11(6): 549-557.

[6] Griebel S M, Brandstein M S. Microphone array source localization using realizable delay vectors[C]. Applications of Signal Processing to Audio and Acoustics, 2001 IEEE Workshop on the. IEEE, 2001:71 - 74.

[7] W.R.Hahn. Optimum signal processing for passive sonar range and bearing estimation [J]. J. Acoust. Soc. Am., 1975, 58 (1): 201-207.

[8] Shen C T, Yu H. Time-delay alignment technique for a randomly distributed sensor array[J]. IET Communications, 2011, 5(8):1068-1072. 